\title{
Design and Fabrication Considerations for Three Dimensional Scaffold Structures
}

\author{
Mazher lqbal Mohammed Parminder Singh Badwal lan Gibson
}

\section{DOI: 10.18502/keg.v2i2.604}

\begin{abstract}
Porous three dimensional structures have seen extensive investigation among design engineers for a wide range of novel applications. The fabrication of such designs would not be possible using traditional manufacturing approaches owing to the dimensional intricacy of such structures, but have now become a distinct possibility owing to the maturity of 3D printing technologies. In this study, we have examined the creation of novel unit cells from mathematic surface renderings as a basis for creating tailored porous structures, before realising the final designs through Fuse Deposition Modelling (FDM) 3D printing. We examined the use of Gyroid and Schwarz primitive $(P)$ surfaces to create novel unit cells not typically found in design software libraries. We then transpose these structures into several test geometries comprising a cylinder, cuboid and tetrahedron, which will adequately test limits of design and fabrication in regular and irregularly shaped structures. It was found that the porosities of the resulting models could be adjusted through discrete dimensional changes in the unit cell and digital wrapping procedures. It was also found that models could be fabricated using FDM printing to a minimum pore diameter of approximately $1 \mathrm{~mm}$ with a high degree of accuracy and repeatability. Ultimately this work will provide guidance to engineering's when creating porous structures and could find usefulness in applications where optimal material usage versus porosity are required, such as in high throughput 3D fluidic applications, such as heat exchangers and tissue engineered structures.
\end{abstract}

\section{Main Text}

\section{Introduction}

Highly controlled micro/macro porous architectures have seen extensive investigation among design engineers in applications ranging from light weight structures for automotive and aeronautical designs; porous structures for tissue engineering [1]; and general aesthetics for artistic purposes [2]. Additive manufacturing techniques have now begun to enable the fabrication of such designs owing to the layer by layer manufacturing approach to realise the final object [3]. Arguably, designs incorporating high density micro/macro pore architectures are the defining element which unlocks the true power of additive 
manufacturing, as fabricating such objects is currently impossible using traditional manufacturing techniques [4]. This synergy of intricate design and additive manufacturing is leading to a paradigm shift in what is now possible in manufacturing terms, with many exciting innovations currently been developed in fields such as implantable medical devices [5] and advanced light weight designs with tailored mechanical properties [6].

A repeating unit cell is at the core of porous designs, from a simple hollow cubic structure to more intricate, nature inspired, Gyroid structures [7, 8]. Despite the ready rendering of such structures, there remains an inability to accurately predict the mechanical strength and the porosity of the final design. Porosity is perhaps the single most influential factor, dictating not only the mechanical strength of a model, but other critical features such as fluidic dynamics and material consumption. Therefore control over the design parameters influencing a models porosity are of fundamental interest if such structures are to be optimised for a particular application.

This study aims to develop a robust procedure to convert mathematical surface renderings into unit cells, which form the repeating unit within intricate porous structures. We then assess the design elements to successfully realise such devices using additive manufacturing. A summary of the proposed process can be seen in Figure 1. As an example, we detail the design procedures involved in rendering a Gyroid and Schwarz P unit cell, while exploring design considerations when transposing the Gyroid unit cell through uniform and non-uniform structures. Such work will prove invaluable for designers wishing to implement porous structures for advanced designs such as part light-weighting, optimised material consumption during manufacturing and specialised applications such as tissue engineering.

\section{Methodology}

\subsection{Unit cell design}

In this study Gyroid and Schwarz P unit cells were examined as the basis for the overall scaffold lattice structure. To create the unit cells we initially began with a mathematical rendering program to create a 2D surface profile, which was then manipulated to form the 3D unit cell. Surface profiles were realised using the open source software MathMod 4.1

(https://sourceforge.net/projects/mathmod/) and output as a polygon wave front OBJ file. Resulting files were then processed using 3-matic (Materialise, Belgium) through a combined process of wrapping and/or boolean subtractions from a parent volume structure, to create the final unit cell.

\subsection{Scaffold Design}

Scaffolds were created using Magics (Materialise, Belgium) whereby a unit cell structure can be transposed at a fixed geometry through a wider 3D model. To evaluate the design criteria for the unit cell integration, three distinct 3D model scaffold shapes were investigated to examine the design criteria under ideal and more challenging design circumstances. Shapes investigated comprised 
of cubic (20x20x20mm), cylindrical (height $25 \mathrm{~mm}$ diameter $25 \mathrm{~mm}$ ) and tetrahedral (square base $20 \times 20 \mathrm{~mm}$ and height $20 \mathrm{~mm}$ ) structures. For the final scaffold structures Gyroid unit cell sizes of 2, 3, 4, and 5mm were examined, resulting varying porosities and boundary continuities in the respective scaffolds.

\subsection{D printing}

In this study a FlashForge Dreamer system was used as the principal FDM printer, which operates using $1.75 \mathrm{~mm}$ filaments, and is open source allowing for complete control of the operational parameters during printing. All scaffolds were printed in ABS plastic using a layer thickness of $0.2 \mathrm{~mm}$, nozzle temperature of $225^{\circ} \mathrm{C}$ and printer bed temperature of $105^{\circ} \mathrm{C}$.

\section{Results}

\subsection{Unit cell Rendering}

The two unit cells employed in this work were chosen specifically to highlight two distinct methodologies for unit cell creation using mathematical surface renderings. The Schwarz P surfaces allow for the unit cell to be rendered directly from the surface model, whereas the Gyroid structures are found in the inverse form and require a Boolean subtraction approach to produce the final unit cell.

When forming the original unit cell from the mathematic formulation, consideration must be paid to the initial boundary conditions to produce a surface structure with a spatial terminus capable of forming a seamless repeating unit. This is required so as to preserve the integrity of a wider scaffold when the unit cell is transposed into a respective model. Schwarz $P$ structures were formed using the formulation of $F(x, y, z)=,\cos (x)+\cos (y)+\cos (z)$ [9], with the boundary conditions of -3.2 to 3.2 for $x, y$ and $z$. Gyroid structures were formed using the formulation of $F(x, y, z)=\cos (x)^{*} \sin (y)+\cos (y)^{*} \sin (z)+\cos (z)^{*} \sin (x)[6]$, with the boundary conditions of $-\pi$ to $\pi$ for $x, y$ and $z$. Once the respective surfaces were rendered, they were imported into 3-matic and wrapped using a gap closing distance of 0.1 and smallest detail parameter of 0.05 , producing a structure with a wall thickness of approximately $0.3 \mathrm{~mm}$. At this point the Schwarzpoly unit cell was ready for use, however the Gyroid unit cell is in an effective inverse form and required a Boolean subtraction from a solid object encompassing the same volume as the complete cell (square $3 \times 3 \times 3 \mathrm{~mm}$ ). Following the subtraction the final Gyroid unit cell was ready for use in the scaffold. Figure 2a) illustrated the digital form of the Schwarzpoly, inverse Gyroid and final Gyroid structures.

\subsection{Scaffold design}

Once the unit cells had been formed, they were scaled to be $2 \times 2 \times 2 \mathrm{~mm}$ at their widest points in the $x, y$ and $z$ directions. The unit cells were then uploaded into the structure library for Magics, which allows for the rendering of the unit 
cell into a given shape, at a desired unit size. From within the Magic software the Gyroid and Schwarzpoly unit cells were translated into a $x=y=z$ unit of 2, 3, 4,5 and $6 \mathrm{~mm}$ within the three scaffold shapes examined. Once this operation was completed, it was found that there were still slight overlap issues at the boundary of each unit cell, which may cause build issues when 3D printing. To remedy this an additional wrapping function was performed.

When constructing the scaffold, consideration must be paid to the outer contour of the model, in particular for instances of partial rendering of the unit cell to low thickness tolerances. An example of this can be seen in figure 2b), where the highlighted region of the outer contour of the cylindrical scaffold is of a thickness of approximately $100 \mu \mathrm{m}$, which would be too thin to be adequately printed by the FDM printer and would likely break off from the wider scaffold. This highlights the importance when constructing scaffolds to perform robust thickness analysis of the wider structure to ensure the integrity of the model relative to the tolerances of the print resolution.

Wrapping of the scaffold structure was performed using 3-Matic and allowed for the overall model to be one continuous part. Wrapping would typically result in an increase in the thickness of the overall scaffold model and so to minimise, was performed using the smallest detail threshold of $0.1 \mathrm{~mm}$, resulting in a uniform increase in thickness of $0.071 \mathrm{~mm}$. Feasibly, a smaller threshold could have been employed, however, for scaffold of unit cell size $<5 \mathrm{~mm}$, would have taken computer power well beyond our current capacity and so this threshold was considered the best compromise. Figure 2c) illustrates an example of a final scaffold model, where thickness analysis and wrapping has been performed.

Following wrapping and saving as an STL, several of the models generated were relative large in size and would be unsuitable for use within the 3D printing software, owing to issues during the slicing phases. To resolve this a procedure to reduce the triangle count was also performed. To achieve this the wrapped models were input into Magics were a triangle reduction using a smallest detail of $0.1 \mathrm{~mm}$, maximum angle of $10^{\circ}$ over 5 iterations was performed. It was found that the files size was reduced on average by an order of magnitude, while also retaining the major details of the scaffold.

\subsection{Scaffold printing}

Once the scaffold models were completed they were printed on the FDM printer in ABS. When printing porous structures using a single material and without soluble supports, it is important to turn the support material off, as this would likely block the pores and be extremely difficult to remove in smaller pores sizes. Despite the supports being off, the designs were believed to be self-supporting in their design and so minimal printing issues were anticipated. Figure 3 shows images of the printed porous structures for each of the examined model variants. It can be seen that the models could be produced with a high degree of precision, realising pore sizes as small as $1 \mathrm{~mm}$. Generally, FDM printed models are creating using a raft material to help avoid issues with warpage during thermal relaxation of printed polymers. However, 
in improving the reproducibility of the models during printing of the initial base layers, when removing the raft minor damage occurred to models with small pore sizes $(<2 \mathrm{~mm})$. This results in one to two layer patches shearing off the bulk of the model. This was believe to occur in the smaller pore structures due to the reduced thickness of the branches within the unit cell, resulting in the a weaker substructure in the scaffold. This problem could be avoided printing without a raft, however all attempts resulted in misprints and so we believe a pore size of $3 \mathrm{~mm}$ is the smallest printable geometry with the given system which preserves the integrity of the overall model.

\section{Conclusion}

In this study we have demonstrated a technique for the conversion of surface renderings into unit cells for use in creating porous structures. We have found that this is possible for two demonstration surface profiles of Gyroid and Schrwarzpoly surfaces and potentially could be applied to a near infinite number of other surface renderings. We also outline a boolean subtraction methodology by which to create negative unit cells from a given surface profile, resulting in unit cell with reduced surface areas, increased porosity and reduced material consumption when printed. The final unit cell can be readily transposed through a variety of model structures to form a given scaffold. When considering the boundary structure, for non-divisible whole number ratios of the model length divided by unit cell and/or irregular boundary shapes, consideration must be made as to what coordinate to begin the repeating unit cell to form the scaffold. Ultimately, we present a technique for creating a near limitless number of unit cell structures from surface renderings and demonstrate the efficacy in creating robust 3D printed scaffolds. This work will prove highly useful when attempting to create advanced structures amendable to 3D printing with adjustable porosities, which will readily find application in areas such as part weighting optimisation, creating structures with adjustable mechanical properties, or tissue engineering applications. 